\title{
Aplicación de la terapia de modelado en la prevención del inicio al consumo de la droga
}

\section{Diego Macía **}

\author{
Universidad de Murcia
}

$\mathrm{E}$

$\mathbf{N}$ nuestro trabajo, pretendemos desarrollar un método preventivo del inicio al consumo de la droga, por medio del aprendizaje observacional, destinado a adolescentes, grupo de la población particularmente expuesto a la farmacodependencia.

En la actualidad el fenómeno de las drogas se presenta con unas características que lo hacen cada vez más preocupante. Para Kramer y Cameron (1975), la inquietud y preocupación que se produce ante el fenómeno de la droga puede deberse principalmente a:

1. El aumento del número de personas que consumen drogas desaprobadas por la sociedad.

2. Su utilización por los jóvenes.

3. El consumo de drogas poco conocidas fuera de las regiones en donde su consumo era tradicional y empleo de otras nuevas.

4. La utilización de la droga en todas las clases sociales.

El consumo de drogas, legales e ilegales, es en nuestro país un tema en torno al cual existe una gran preocupación social, totalmente justificada a la luz de las encuestas y los datos de que se dispone en la actualidad sobre dicho consumo.

Por lo que respecta al uso de distintas drogas, tanto denominadas legales como ilegales, las dificultades para un sondeo enidemiológico son obvias. No es posible exponer con detalle la situación, sin embargo vamos a considerar una serie de datos que nos pueden hacer $\operatorname{com}_{\rightarrow 5}$. prender la magnitud del problema que estamos tratando.

Al hablar del consumo de drogas es muy importante estudiar o considerar por separado cada una de las sustancias.

En una encuesta de Cidur/Edis (1980), realizada con una muestra de 1.600 jóvenes de edades comprendidas entre $12 \mathrm{y}$ 24 años, a la pregunta: « $¿ \mathrm{Ha}$ probado o usado algún tipo de droga?» (se refiere a las ilegales) aparecen los siguientes resultados:

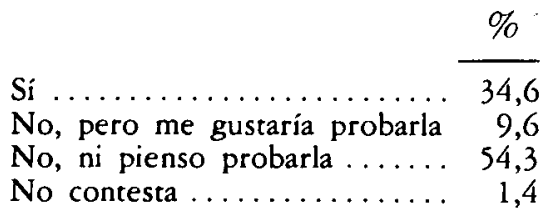

Con respecto al alcohol, la «Memoria del grupo de trabajo para el estudio de

- El presente trabajo constituye un resumen de la Tesis Doctoral defendida por el autor en la Universidad nurớnuma de Múd-:

** Dirección del autor: Facultad de Filosofía y Ciencias de la Educación, Sección de Psicologia. Ũniversi= dad de Murcia. 
los problemas derivados del alcoholismo y del tráfico y consumo de estupefacientes» del Ministerio de la Gebérnàción, en 1975, reconocía la existencia dé un 2,5 por 100 de alcohólicos y de un 5 por 100 que eran bebedores habituates en cantidades preocupantes. (En Mendoza y Vega, 1980).

En relación al tabaćo, ségún datos de la propia: Tabacalera,: S. A. (en Cáritas Española-Edis, 1981): existẹn en España 12,5 millones de fumadorrés:

Respecto al uso de psicofármacos en España, las cifras son ciertamente muy elevadas, según Freix̌a, Sölét y colaboradores (1981), en 1.979 se consumieron 159 millones de enväses de psicofármacos.

La Organización* Mundial de la Salud, Kramer y Cameron (1975), reclama la necesidad de técnicas avanzadas para influir en los grupos. de la población particularmente expuestos (v. g., adolescentes, etc.). Para la OMS junto a medidas sociales y legales, las medidas educativas son la base de la labor preventiva. Medidas educativas que deben tener por objetivo un cambio en las actitudes hacia las drogas y su consumo.

Para Kramer y Cameron (1975) los diversos métodos preventivos de la farmacodependencia pueden clasificarse en tres apartados: 1) reglamentación legal, encaminada a restringir las posibilidades de procurarse drogas; 2 ) medidas educativas destinadas a informar al ciudadano sobre las drogas y a influir en las actitudes relativas al uso de las mismas, y 3.) medidas sociales en favor de los grupos expuestos.

Goodstadt (1978) indica que hay dos grandes modelos de programas de educación sobre las drogas: en el primero se supone que, aumentando los conocimientos o modificando las actitudes, se logrará al final cambiar los hábitos de consumo de drogas. En el segundo, se parte de la hipótesis de que inculcando o reforzando determinados valores se modificarán los hábitos.

Los primeros programas educativos propuestos para la prevención del consumo de la droga, adolecieron de un exceso de confianza en el hecho de que la información que se pudiese proporcionar, sobre los efectos negativos del abuso de las drogas, harían tomar a los individuos decisiones fundadas ante este problema. La premisa básica era la de que cuando se conocieran hechos concretos se cambiaría la actitud hacia la droga.

Estos primeros programas preventivos, basados primordialmente en el uso de información biológica y farmacológica, fueron criticados por quienes suponían que en ellos se ignoraban las motivaciones psicológicas y sociales de la conducta del toxicómano.

Se plantearon entonces programas educativos de corte psicosocial que intentaron pother al descubierto factores «causales» subyacentes. Al mismo tiempo se reclama la necesidad del compromiso activo de los participantes en los programas educativos y la solución de sus problemas.

Pero las limitaciones de estos programas, a nuestro modo de ver, radicaban en que su base de sustentación estaba en una hipotética fuerza de voluntad, en una capacidad de autorrealización del individuo y en su capacidad de desarrollarse en. las dimensiones social, emocional y espiritual.

En nuestro trabajo, que se enmarca dentro del paradigma de 1 â Psicología Comunitaria, la prevención es entendida como un conjunto de estrategias que proporcionan a los sujetos las experiencias de aprendizaje de competencias que le permiten prevenir el desajuste.

Como afirman Costa y López (1982) el Modelo de Competencia asume plenamente el derecho que toda persona tiene de poder desarrollar competencias o habilidades que le capaciten para prevenir y resolver problemas y manejar el stress de una manera adecuada. Este modelo apuesta de forma terminante por la fuerza de la habilidad (frente a la hipotética fuerza de voluntad) o de competencia y se empeña en fomentar todas aquellas estrategras de intervención con las que se ponga a disposición de la persona la oportunidad de aprender a ser competente.

En nuestro trabajo no se pretende modificar los hábitos de consumo de los ya iniciados, no se espera que nuestro modelo de intervención disminuya el uso de las drogas en los jóvenes que ya consumen, nosotros nos centramos en el inicio, 
intentamos prevenir el comienzo del consumo, incidimos en la prevención primaria.

Tras un reconocimiento de los principales factores psicosociales que son causa en la mayoría de los casos del inicio a la drogadicción, pretendemos dotar de habilidades suficientes a los jóvenes con el fin de que puedan hacer frente a esas situaciones o problemas.

Por otra parte, somos conscientes de que en un programa de intervención para grupos es imposible abarcar todas las posibles causas que, individualmente en algunos casos, llevan al joven al consumo de drogas. Aspectos tan importantes como el fracaso escolar, falta de comunicación educador-educando, problemas familiares y un largo etcétera quedan fuera por fuerza de nuestra área de actuación.

$\mathrm{Ni}$ que decir tiene que comprendemos la necesidad de otros tipos de medidas preventivas simultáneas, legales y sociales, que junto con la formación y el aprendizaje de habilidades completarían la labor preventiva.

Así, uno de los objetivos de nuestro trabajo era comprobar en qué-medida la aplicación de un programa educativo puede provocar un cambio en la actitud hacia la droga y su consumo. Con este fin en uno de los grupos experimentales se aplicó un programa.eduçativo.

En este grupo se daba una información que tenía por objetivo: a) Suministrar unos conocimientos sobre las distintas drogas y sus efectos. b) Poner al alcance de los jóvenes los conocimientos que se poseen sobre las causas de iniciación y circunstancias de consumo. c) Proporcionar valores, creencias y actitudes que se opusiesen al abuso de las drogas. d) Inducir en los jóvenes soluciones alternativas capaces de responder a sus necesidades $y$ aspiraciones.

Se trata de un programa educativo en el que la información no se concibe como la mera transmisión de conocimientos, o no se limita sólo a la información, sino que persigue la formación de los jóvenes.

Cabe pensar que este tipo de programa es válido para conseguir un cambio en la actitud. Sin embargo, nuestras dudas se centraban en la repercusión que pudiese tener sobre el comportamiento futuro de los jóvenes.

La exhortación, la persuasión moral y el hecho de apelar al poder del sujeto, su formación e información, pueden provocar un cambio en la actitud y posiblemente en la intencionalidad del comportamiento. Pero esto, no entrena al sujeto a resolver una situación problemática, ni le capacita para afrontarla por medio de un bagaje de habilidades que sin duda le serán útiles y necesarias.

Pö este motivo consideramos la necesidad de contrastar los resultados de-este primer grupo con los de un segundo en el que junto a ese programa educativo a los sujetos se les permitiese observar la puesta en práctica de comportamientos coherentes con el cambio de actitud y representarlos (Grupo II, programa educativo-más modelado y role-playing).

Se pretendía que los sujetos pudiesen observar que ciertos comportamientos se podían dar (por ejemplo: resistirse a las presiones del grupo o dar argumentos enun grupo sobre los efectos nocivos de una droga determinada). Se partía del supuesto de que la información-educación sưlà pơdía no ser suficiente sirno se acompañaba de un aprendizaje en habilidades comportamentales.

La utilización de una técnica de modelado fue por considerar que estas técnicas basadas en el aprendizaje vicario pueden ser una herramienta útil, tanto a utilizar en el cambio de actitudes, como en el aprendizaje de conductas sociales (Bandura, 1982).

Un tercer grupo sirvió para comprobar si sólo la oportunidad de observar modelos que representan comportamientos contrarios al uso y abuso de las drogas y representar esos papeles, era suficiente para promover un cambio de actitud y el aprendizaje de esas habilidades comportamentales.

\section{HIPOTESIS}

Las hipótesis de las que partimos en la presente investigación son:

1. La actitud hacia la droga cambiará con ia puesta en niartita da programas educativos. 
2. El programa educativo modificará el nivel de conocimientos sobre las drogas.

3. El programa educativo no cambiará los hábitos relacionados con el consumo de drogas.

4. Un entrenamiento de modelado más ejercicios mediante la técnica de role-playing facilitará el aprendizaje de comportamientos que ayuden a la prevención del inicio al consumo de drogas.

5. U'n programa educativo más modelado y la técnica de role-playing es más adecuado para el cambio de actitudes y la adquisición de habilidades comportamentales que cualquiera de los dos por separado.

6. Un programa educativo más modelado y la técnica de role-playing no va a cambiar los hábitos de consumo de los sujetos ya iniciados en las drogas.

\section{METODO}

\section{Muestra empleada.}

Los sujetos fueron 136 alumnos estudiantes de primero de Formación Profesional de un centro en la provincia de Alicante.

\section{Pruebas utilizadas.}

Con objeto de evaluar nuestros procedimientos, se recogieron una serie de datos con la aplicación de las siguientes pruebas:

a) «Cuestionario de hábitos de consumo de droga». Se aplicó con el fin de recoger de la forma más precisa y exacta información sobre el consumo de todas las clases de droga.

b) «Cuestionario sobre el contexto de iniciación». A fin de disponer de una información objetiva y precisa sobre el contexto físico y social que rodeó la primera experiencia con la droga, así como conocer las consecuencias que produjeron el consumo, tanto a nivel de experiencia propia como de los demás con respecto a la persona que lo probó.

c) «Escalas de actitudes». Para la construcción de las escalas utilizamos la técnica de Likert de calificaciones sumadas. Se construyeron un total de nueve escalas, tres para tabaco (componentes: cognitivo, afectivo y comportamental), tres para alcohol y tres para psicofárma$\cos \mathrm{y}$ drogas ilegales.

d). "Cuestionario de información". Se aplicaron tres, alcohol, tabaco y psicofármacos y drogas ilegales. Tenían por objeto evaluar el conocimiento de los sujetos sobre cada tipo particular de droga.

Por otra parte, con el fin de, en su caso, proceder a controlar las variables: inteligencia, medio socio-cultural y la variable de personalidad: adaptación familiar, a la salud, social y emocional, se aplicaron:

a) «Encuesta socioeconómica».

b) Test de Inteligencia General 1.

c) Cuestionario de adaptación para adolescentes de Bell.

\section{Procedimiento}

Aplicación inicial.

En una fase previa al inicio del programa educativo y a las sesiones de modelado, se aplicaron a todos los sujetos, incluidos los del grupo de control, toda la anterior serie de cuestionarios y pruebas psicológicas.

A continuación se distribuyeron los cursos al azar a cada futuro grupo, y a los alumnos de cada grupo, también al azar, a cada uno de los investigadores.

Todo el material recogido se corrigió inmediatamente con el fin de tener un conocimiento preciso de los sujetos con respecto al tema a tratar. Iniciándose entonces en cada grupo el progama de trabajo.

Aplicación del entrenamiento.

Con el Grupo I (Programa Educativo) se programaron siete sesiones, una por semana, de una hora de duración. Cada una de estas sesiones consistía en una charla inicial seguida de un coloquiodiscusión, en la que los sujetos aportaban todo el material recogido, ya que cada semana se les adelantaba el tema a tratar al día siguiente. Se intentó, dado que el grupo era reducido, que se diese el máximo de participación en las discusiones.

Los temas de las siete sesiones programadas fueron:

1. Concepto de droga, tipos y efectos. 
2. Incidencia, la iniciación y sus causas.

3. El marco de iniciación y la presión del grupo.

4. Efectos psico-sociales de las drogas.

5. Psicología social de los grupos.

6. Personalidad, proyecto de vida e ideologia.

7. Juventud y tiempo libre.

Los objetivos particulares de cada una de las sesiones fueron:

a) Que adquieran unos conocimientos básicos sobre las drogas y conseguir una actitud favorable al mantenimiento de la salud.

b) Prevenir la curiosidad en el inicio. Advertir del peligro que entraña el transigir, aun siendo para probarlo..

c) Que reconociesen la importancia que tiene la presión del grupo a la hora de tomar una decisión.

d) Que comprendiesen la importancia de rechazar las gratificaciones inmediatas y sentar las bases para alcanzar metas futuras más importantes.

a) Se pretende que reconozcan las situaciones que comúnmente terminan con el ofrecimiento y que valoren que pueden tomar su propia decisión con absoluta independencia de los demás.

f) Ser fiel a la propia escala de valores y desarrollo del autoanálisis:

g) Descubrir alternativas válidas a las drogas.

Para el Grupo II (Programa Edúcativo más modelado y. role-playing) se programó el mismo número de sesiones y se mantuvieron los mismos temas y los mismos objetivos particulares de cada sesión del Grupo I.

En este grupo, una vez terminada la charla, se daba comienzo al modelado y role-playing. Para esto, de los mismos componentes del grupo, se elegía a algunos sujetos y se les entregaba un papel con el diálogo a representar. Antes de empezar-se les leía un texto breve para: introducir el diálogo y centrar su atención en los aspectos más relevantes de éste.

Se hacian unas siete u ocho represen- taciones, donde los sujetos en uno u otro papel participaban siempre.

Cada representación servía para iniciar una discusión de todo el grupo sobre la charla y lo representado.

En el Grupo III (Grupo de modelado más role-playing) se programaron igual número de sesiones con los mismos temas y objetivos particulares que en los grupos anteriores. En este grupo, el trabajo de cada sesión, se iniciaba directamente con la lectura del texto breve que en el Grupo II preparaba la intervención de los modelos y el role-playing.

Una vez finalizadas las sesiones de trabajo en los tres grupos, a los diez días se volvieron a aplicar las «Escalas de actitud» y los «Cuestionarios de información» a los cuatro grupos.

Posteriormente, transcurridos seis meses se efectuó un seguimiento, en el que se aplicaron las «Escalas de actitud» y el "Cuestionario de hábitos de consumo" a los cuatro grupos.

\section{RESULTADOS}

El procedimiento estadístico ha consistido en:

a) La realización de un análisis de varianza para comparar los resultados en inteligencia, adaptación y medio-: sociocultural de los cuatro grupos.

Tenía por finalidad comprobar si a pesar de ser grupos supuestamente semejantes, diferian significativamente entre ellos. En ningún caso se encontraron diferencias significativas entre los grupos, por lo que estas variables no fueron tenidas en cuenta en los análisis estadísticos posteriores.

b) Análisis de covarianza con un diseño de dos factores (grupos e investigadores) para cada una de las variablès (alcohol, tabaco y otras drogas) del «Cuestionario de información", con las puntuaciones obtenidas antes y después del tratamiento.

Para cada una de las variables (alcohol, tabaco y otras drogas) aparecen diferencias significativas entre los grupos [alcohol: $\mathbf{F}(3,111)=2,93(\mathrm{p}<0.05)$; tabaco: $f(3.111)=5.47(p<0.01)$; otras drogas: $\mathrm{F}(3,111)=23,44(\mathrm{p}<0.01)]$. 
No siendo significativa ni la interacción ni el efecto de los investigadores.

La comparación posterior de las medias ajustadas de los grupos nos mostró que dichos resultados eran debidos a las diferencias entre: el Grupo I y el Grupo IIF, el Grupo.I y el IV, el Grupo II y el III, y el Grupo II y el Grupo IV. Es decir que el cambio se produce en los dos grupos en que se había proporcionado información.

c) Análisis de covarianza, con un diseño de dos factores (grupos e investigadores) para cada una de las variables (alcohol, tabaco y otras drogas) y para cada uno de los componentes (cognitivo, afectivo y comportamental), con las puntuaciones obtenidas antes y después del tratamiento en las «Escalas de actitudes».

Aparecen diferencias significativas $\mathbf{F}$ (3) $95)=4,2(\mathrm{p}<0.01)$, para la variable alcohol en el componente comportamental. Al realizar la comparación entre las medias ajustadas de los grupos obtuvimos significación estadística al comparar los grupos: I y II; I y III; II y IV; y III y IV. En los grupos- con modelado y role-playing.

En lo referente a la variable tabaco el análisis de covarianza no arrojó diferencias significativas.

Con respecto a las otras drogas, el análisis de covarianza muestra una diferencia significativa entre los grupos en el componente afectivo, $F(3,95)=4,57$ $(\mathrm{p}<0.01)$. Al comparar las medidas ajustadas de los grupos observamos que dicho resultado es debido a la diferencia entre los grupos: I y III; I y IV; II y III; Il y IV. Es decir, se produce un cambio con la aplicación del programa educativo.

También en el componente comportamental se observa una diferencia significativa entre los grupos, $F(3,95)=7,04$ ( $<<0.01)$., dicha diferencia es debida a la existente entre los grupos: I y II, I y III, II y IV, III y IV. Cambian los grupos con modelado y role-playing.

d) Análisis de varianza con los resultados a las «Escalas de actitud» en el seguimiento para cada una de las variables y para cada uno de los componentes. De las tres componentes de la variable alcohol sólo aparecen diferencias significativas en el comportamental $F(3,102)=3,98$ $(p<0.01)$. Dicho resultado es debido a la diferencia significativa existente entre el resultado del Grupo II respecto a todos los demás.

En la variable tabaco no existen diferencias significativas.

En.lo referente a la variable otras drogas, no existen diferencias significativas entre los grupos en el componente cognitivo, pero sí en los otros dos. En el componente afectivo, la diferencia $F(3,102)=$ $=4,3852(\mathrm{p}<0.01)$ es debida a la existente entre los grupos: I y IV, - II y IV; cambia con el programa educativo. En el componente comportamental la diferencia $F(3,102)=4,42(p<0.01)$ es debida a la existente entre los grupos II y III con I y IV. Es decir, de los grupos con modelado y role-playing.

e) Significación de diferencias de proporciones entre los grupos con los resultados al «Cuestionario de hábitos de consumo» antes del tratamiento, y el mismo procedimiento con los resultados a dicho cuestionario obtenidos en el seguimiento a los seis meses. En lo relativo al análisis estadístico realizado; no aparecen diferencias significativas.

\section{DISCUSION}

En nuestro trabajo uno de los primeros objetivos era comprobar en qué medida era posible modificar la actitud de los jóvenes hacia las drogas con la puesta en marcha de un Programa Educativo. Esta primera hipótesis queda en nuestra investigación confirmada parcialmente, dado que ésta es cierta para el componente afectivo en el caso de los psicofármacos y drogas ilegales, donde aparecen diferencias significativas entre el Grupo I (Programa Educativo) y el Grupo IV (Control). Resultados que se mantienen en el seguimiento.

Estos resultados, vienen a confirmar que la puesta en práctica de programas educativos, son útiles para el cambio de actitudes. Hecho éste que ha sido observado en distintas revisiones sobre los programas educativos puestos en práctica (Goodtandt, 1974, 1978; Brawn y Klein, 1975; Freixa Soler y otros, 1981).

Con respecto al componente cognitivo de la actitud, donde no aparecen dife- 
rencias significativas, esto puede ser debido a dos motivos. El primero al hecho de que al enunciar y proponer los objetivos del Grupo I, se hizo hincapié en que no se convirtiera la información suministrada en la que es propia de los grupos denominados de "recurso al miedo». El segundo motivo estriba-en que inicialmente los sujetos en el componente cognitivo obtienen ya una puntuación elevada, es decir, lo perciben correctamente.

Nuestra ségunda hipótesis consiste en que: el programa educativo cambia el nivel de conocimientos sobre las drogas, queda confirmada dado que tanto para el alcohol como para el tabaco y las otras drogas, aparecen diferencias significativas en los "Cuestionarios de información" del Grupo I y el III y IV. Y. del II con el LI y el IV.

La tercera hipótesis, consistente en que el programa educativorro cambia los hábitos posteriores relacionados con el consumo de drogas, queda confirmada dado que los resultados del análisis estadístico efectuado con los datos obtenidos en el "Cuestionario de hábitos de consumo" antes del tratamiento, así como en el seguimiento, no aparecen diferencias significativas entre el Grupo I y el IV, ni en el número de sujetos que consumen droga ni en las cantidades consumidas.

Este resultado obtenido en nuestra investigación viene a corroborar lo expuesto en experiencias anteriores, donde queda de manifiesto la falta de evidencia de que cambiando las actitudes e inculcando ciertos valores se cambian los hábitos relacionados con las drogas (Mendoza, en Freixa, Soler y otros, 1981).

La cuarta hipótesis formulada por nosotros en el sentido de que: el modelado más role-playing hace posible el aprendizaje de comportamientos que ayudan a la prevención del inicio al consumo de la droga; no ha podido ser comprobada. Si bien es importante constatar que en el componente comportamental de la actitud, tanto en el apartado alcohol como en el de otras drogas aparecen diferencias significativas entre el Grupo III y el Grupo I, y entre el Grupo III y el Grupo IV. Lo que indica una actitud más favorable a la realización de determinados comportamientos que ravorecerán ia posibilidad de resistirse al inicio al consumo.
Nuestra quinta hipótesis: un programa educativo más modelado y role-playing es más adecuado para el cambio de acritud y la adquisición de habilidades comportamentales que cualquiera de los dos por separado; queda confirmada de forma parcial en la medida en que en el Grupo II los cambios son significativos en el componente comportamental de la.actitud hacia el alcohol, en el componente afectivo y comportamental en la variable psicofármacos y otras drogas. Sin embargo no se - ha podido demostrar con respecto a los hábitos de consumo. Debiéndose posiblemente al reducido período de tiempo del seguimiento.

Nuestra sexta y última hipótesis: un programa educativo más modelado y role-playing no va a cambiar los hábitos de consumo de los ya iniciados; queda confirmada al no encontrar diferencias significativas entre los grupos II y el de Control.

Con relativa frecuencia se cuestiona la posible eficacia de los programas educativos en su incidencia sobre los hábitos de consumo, y esto con razón, pues muchos de estos programas se marcan como objetivo el disminuir el uso de las distintas

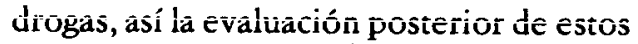
programas se hace en función de este criterio, cuando parece que estos programas sí están indicados es para prevenir el inicio, pero dificilmente podrán eliminar o disminuir el consumo de los ya iniciados.

Un aspecto importante que sobresale de los resultados es sin duda el que se refiere al tabaco, donde en ninguno de los grupos aparece el más mínimo cambio. Esto hace suponer que este tipo de droga por sus características particulares, no permite ser tratado conjuntamente con las demás drogas.

Por otra parte habría que señalar que en los sujetos con que se ha trabajado, de antemano había ya una actitud muy favorable al no consumo, hecho que puede estar enmascarando la efectividad del tratamiento, ya que hace más difícil observar después cambios significativos, al presentar la gran mayoria una actitud inicial objetiva y favorable al no consumo.

De este modo puede haber ocurrido que, si los sujetos perciben adecuadamente las drogas, sólo en las jlegales se

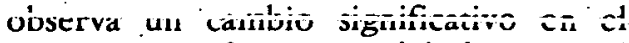
componente afectivo, es debido a que el $-4-\bar{j}$ 
grupo de antemano es contrario a las drogas, si bien, no reconocen la posibilidad, ni la conveniencia, de dar en determinados momentos comportamientos que les pueden ayudar a hacer frente al inicio. Aspecto éste que sí cambia significativamente después del tratamiento, apareciendo una actitud más favorable a la realización de determinados comportamientos que favorecerán la posibilidad de resistirse al inicio al consumo.

\section{Referencias}

Bandura, A.: Teoria del Aprendizaje Social. Madrid. Espasa-Calpe, 1982.

BRAWN, E. H., y KLEIN, A. L.: The effects of drug education programs attitude change. Journal of Drug Education, 1975 , vol. 5 , n. ${ }^{\circ} 1,51-56$.

CARITAS ESPAÑola-EDIS: La población española ante las drogas. En Documentación Social, 1981, n. ${ }^{0} 42$. CiduREDis: Juventud y droga en España. Subdirección General de estudios e investigación. Madrid, 1980.

COSTA, M., y LÓPEZ, E.: La Psicologia Comunitaria: un paradigma nuevo. Papeles del Colegio, 1982, n. ${ }^{\circ} 2$, pag. 17-22.

FreIXA, F.; SOlER, P. A., y cols.: Toxicomanias, un enfoque multidisciplinario. Barcelona. Fontanella, 1981.

GoODSTADT, M. S.: Research on methods and programs of drug education. Addiction Research Foundation, Toronto, 1974.

GoODSTADT, M. S.: Alchol and drug education: models and outcomes Health Education Monographs, 1978, 6, 263-279

Kramer, J. F., y Cameron, D. C.: Manual sobre dependencia de las drogas. Organización Mundial de la Salud. Ginebra, 1975.

MENDOZA, R., y VEGA, A.: El papel del educador ante el problema de las drogas. Madrid, 1980. Pablo del Río, Editor.

\section{Resumen}

La presente investigación ba consistido en desarrollar un método preventivo del inicio al consumo de droga, por medio del aprendizaje observacional, destinado a adolescentes. Se pretendia modificar la actitud hacia la droga, asi como dotar de habilidades comportamentales suficientes a los sujetos, con el fin de que pudieran hacer frente a determinadas situaciones, causa, en muchos casos, del inicio de la drogadicción.

La bipótesis principal era que un programa educativo, más modelado y la técnica de rol-playing, cambiará la actitud bacia la droga y facilitará el aprendizaje de comportamientos que ayuden a la prevención.

Los resultados indican un cambio de actitud bacia determinadas drogas y una actitud más favorable a la realización de determinados comportamientos que favorecerán la posibilidad de resistirse a la iniciación al consumo de la droga.

\section{Summary}

The present research study bas consisted in the development of a method to prevent the initiation in drug consumption, through the use of observational learning and addressed to the adolescents. It was intended to modify the attitude towards drugs as well as to provide the subjects with the required bebavioral skills with the purpose of coping with certain situations, which in many cases are causes of initiation in drugaddiction.

The main bypothesis was that an educational programm, plus modelling and role-playing technique would change the attitude towars drugs and would facilitate the learning of behaviors which will belp with the pretention.

The results indicate a change in attitudes toward certain drugs and a more favorable attitude towards performing certain behaviors which facilitate the possibility of ressisting the iniciation of drug consumption.

\section{Résumé}

Cette recherche a essayé de developper une méthode de prevention sur l'initiation à la consomation de la drogue, par moyen de l'apprentissage observationel, dirigé à des adolescents. Il s'agit de modifier l'attitude envers la drogue de même que de douer d'babilités comportamentales valables aux sujets à fin qu'ils puissent aborder certains situations qui, parfois, sont à l'origine de la consommation de drogue.

L'bipotbèse principale c'est qu'un programe educatif plus les techniques de modelling et role-playing va changer l'attitude vers la drogue et va faciliter l'apprentissage de comportements qui puissent contribuer à la prevention.

Les resultats montrent un changement d'attitude vers certains drogues et une attitude plus favorable à la mise en oeutre de certains comportements qui vont augmenter la possibilité de resister à l'initiation à la consommation. 